$\mathbb{P}$ periodica polytechnica

Chemical Engineering

$53 / 2$ (2009) 49,54

doi: $10.3311 /$ pp.ch.2009-2.03

web: http://www.pp.bme.hu/ch

(c) Periodica Polytechnica 2009

RESEARCH ARTICLE

\section{Preparation and characterization of Thioflavin T doped silica nanoparticles}

\author{
Balázs Söptei / Péter Baranyai / Attila Lajos Kovács / Zoltán Hórvölgyi
}

Received 2009-05-27

\begin{abstract}
Fluorescent silica nanoparticles with diameters of about $300 \mathrm{~nm}$ were synthesized based on Stöber's method, using Thioflavin T as fluorescent co-reagent. The particles were characterized via transmission electron microscopy and fuorimetry measurements. Fluorescence intensity of the sols was ten times higher than that of the ethanol phase solutions of Thioflavin T. Release of dye molecules in stable alcosols was investigated by measuring UV-Vis absorbance spectrum of the supernatant. To try an alternative route, we investigated accumulation of dye molecules in native silica particles. No release effect was detected, and slow accumulation was observed. Water contact angles of the particles were assessed from analyzing the Langmuir films, and were found to be $18^{\circ}$, very similar to native silica particles. Langmuir-Blodgett films of the particles were deposited on a glass substrate and were examined via UV-Vis spectrophotometry, fluorimetry and scanning electron microscopy. Presence of the film was revealed; the particles formed a continuous, well-packed monolayer.
\end{abstract}

\section{Keywords}

fluorescent nanoparticles $\cdot$ Thioflavin $T \cdot$ Stöber silica $\cdot$ film balance $\cdot$ wettability $\cdot$ Langmuir-Blodgett films

\section{Acknowledgement}

We gratefully acknowledge the financial support of the Hungarian Scientific Research Fund (OTKA CK 78629).

\section{Balázs Söptei}

\section{Péter Baranyai}

Department of Physical Chemistry and Materials Science, BME, H-1525 Budapest, P.O.B. 17, Hungary

\section{Attila Lajos Kovács}

Department of General Zoology, Cell Physiology Laboratory, ELTE, H-1528 Budapest, Hungary

\section{Zoltán Hórvölgyi}

Department of Physical Chemistry and Materials Science, BME, H-1521, Budapest, Hungary

e-mail: zhorvolgyi@mail.bme.hu

\section{Introduction}

Thioflavin $\mathrm{T}$ is a biocompatible fluorescent dye. It is frequently applied in detection of $\beta$-sheet proteins, since when it is intercalated with $\beta$-sheets, the dye undergoes a fluorescence shift[1], and this specific interaction can be used for in vitro detection of diseases such as bovine spongiform encephalopathy (BSE) and Alzheimer disease in an early stage [2].

By reason of biological properties and interaction of the mammalian nervous and circadian system, molecules themselves cannot get to the brain carried by the blood, since the blood brain barrier (BBB) limits entrance of several substrates [3]. Modern pharmaceutics give an alternative way to avoid this obstacle. Built into nanoparticles, dye molecules can be delivered and reach through the $\mathrm{BBB}$, and take effect in brain cells. In medical biology, controlled drug delivery is an emerging area in the application of nanoparticles[4], which is now exploited mostly in cancer therapy [5,6].

On the other hand - due to its fluorescent properties -, Thioflavin $\mathrm{T}$ is a potential material for doping signaling particles and fluorescent nanoprobes. These small particles have opportunities of application in several areas, such as medical diagnostics and controlled drug delivery. Fluorescent phenomena can also be used to determine efficiency of drug delivery systems.

Thioflavin $\mathrm{T}$ has been previously integrated into polystyrenePCBA (poly-butyl-2-cyanoacrilate) core-shell [7] and latex [8] nanoparticles, which release the dye under specific conditions.

Although silica nanoparticle synthesis according to Stöber's method [9] is a robust and easy-to-implement technique, Thioflavin $\mathrm{T}$ has not been incorporated into this type of particles. Stöber silica has plenty of advantages, such as spherical shape and narrow size distribution, and low toxicity [10]. It has already been used for synthesizing fluorescein [11] and rhodamin[12] doped fluorescent silica nanoparticles. Previous studies showed that silica particles can be applied for establishing functional layers[13, 14], but - until now - there were no experiments which investigated properties of fluorescent particle layers.

In this study, we describe a method for preparation of fluorescent Stöber silica nanoparticles doped with Thioflavin T, via a 
one-pot synthesis[15]. With this method, advantages of this dye and silica particles can be combined. If silica nanoparticles incorporate Thioflavin $\mathrm{T}$ they could function as signaling probes. Furthermore, we prepared particulate Langmuir and LangmuirBlodgett (LB) films to characterize the synthesized samples.

\section{Experimental section}

\subsection{Materials}

For the synthesis and purification of fluorescent nanoparticles tetraethyl-orthosilicate (> 98\%, GC grade, Merck) (TEOS), absolute ethanol (a. r., Reanal), aqueous solution of ammonia (25\%, a. r., Reanal) and Thioflavin $\mathrm{T}(75 \%$, Fluka) were used. For film balance experiments we used chloroform (ultra-resi analyzed, 99.8\%, J. T. Baker) and distilled water (18.2M $\Omega \mathrm{cm})$ purified with a Millipore Simplicity 185 filtration system.

\subsection{Methods}

\subsubsection{Preparation of fluorescent silica nanoparticles}

Alcosols of fluorescent silica particles were synthesized according to Stöber's method [9]; Thioflavin T was added to the reaction mixture after formation of microphases. The presence of microphases was evidenced by examination of Tyndall phenomenon (observed by naked eye). We used different amounts of Thioflavin $\mathrm{T}$ (see data in Table 1) to prepare silica particles with different fluorescence intensity.

Tab. 1. Amount of Thioflavin $\mathrm{T}$ used for the synthesis of fluorescent silica sols (added to a mixture containing $250 \mathrm{~cm}^{3}$ of ethanol, $10 \mathrm{~cm}^{3}$ of TEOS, and aqueous solution of ammonia)

\begin{tabular}{lc}
\hline Sol & Thioflavin $\mathbf{T}(\mathbf{g})$ \\
\hline FB300B & 0.028 \\
FB300C & 0.056 \\
FB300D & 0.084 \\
FB300E & 0.112 \\
FB300F & 0.140 \\
\hline
\end{tabular}

\subsubsection{Purification of alcosols of fluorescent particles}

Ammonia was removed from the sols by vacuum distillation at $50^{\circ} \mathrm{C}$. To separate the particles from the supernatant containing residual Thioflavin $\mathrm{T}$, we centrifuged sols at 4300rpm for 30minutes, and dispersed the particles in pure ethanol; this step was repeated three times. After purification we determined the solid content of the alcosols from the mass of residual solids after the evaporation of ethanol in a drying oven at $120^{\circ} \mathrm{C}$ for 3hours. As a result of purification, we obtained a pure ethanolic sol of the fluorescent nanoparticles.

\subsubsection{Preparation of fluorescent silica hydrosols}

To prepare hydrosols of the particles, we centrifuged the sols at 4300rpm for 30minutes. After centrifugation, we removed the supernatant and dispersed the particles in distilled water. This method was repeated three times. We assumed that via this technique, the amount of ethanol residues is negligible in hydrosols.

\subsubsection{Particle size measurement}

For transmission electron microscopy (TEM) investigations we deposited the particles on a Formvar film coated copper grid. We used a JEOL JEM-100 CXII transmission electron microscope for the measurements. Electron micrographs were used to determine particle size distribution of the sols. TEM samples were taken from the film of the nanoparticles spread on water surface in a Petri dish.

\subsubsection{Adsorption experiment}

To gain information about the accumulation of Thioflavin $\mathrm{T}$ molecules on the surface or adsorption in the inner part of the pores of silica nanoparticles, we added the dye to an alcosol of unmodified Stöber silica particles, solid content of $16.0 \mathrm{mg} / \mathrm{ml}$. The mixture was stirred in a thermostat at $25^{\circ} \mathrm{C}$. We measured UV-Vis absorbance spectrum of the starting dye solution, and the supernatant after 1, 2 and 7 days later, respectively. For separating the supernatant we centrifuged the sols at $4300 \mathrm{rpm}$ for 30 minutes.

\subsubsection{Release of Thioflavin $\mathrm{T}$ molecules from silica parti- cles}

To obtain information about the dye release from the particles, we stored the prepared and purified fluorescent silica alcosols at room temperature, without agitation. Another part of silica alcosols was exposed to a $50^{\circ} \mathrm{C}$ heat shock for one hour, and was subsequently stored at room temperature. After 7and 14days, sols were centrifuged and the absorbance spectrum of the supernatant was measured with an Agilent 8453 UV-Vis spectrophotometer.

\subsubsection{Fluorimetry measurement}

To obtain quantitative information about the fluorescence of the sols, a Perkin-Elmer Luminescence Spectrometer LS 50 B was used. Before the measurements, solid phase content of each sol was set to $12.0,2.4$ and $1.2 \mathrm{mg} / \mathrm{ml}$, respectively. Emission spectra were obtained with an excitation wavelength of 400nm, a $5 \mathrm{~nm}$ excitation slit, and a $5 \mathrm{~nm}$ emission slit. We gained excitation spectra with an emission wavelength of $480 \mathrm{~nm}$.

Tab. 2. Particle diameter and std. deviance of fluorescent silica sols

\begin{tabular}{lll}
\hline Sol & Mean diameter $(\mathrm{nm})$ & Std. deviance $(\%)$ \\
\hline FB300B & $274 \pm 5$ & 11.31 \\
FB300C & $254 \pm 4$ & 9.06 \\
FB300D & $253 \pm 4$ & 9.88 \\
FB300E & $284 \pm 4$ & 8.80 \\
\hline
\end{tabular}

\subsubsection{Preparation and characterization of particulate Lang- muir films}

For the preparation of particulate Langmuir films a laboratory-built Wilhelmy film balance was used. For the film balance experiments, solid content of fluorescent alcosol FB300E was set to $12 \mathrm{mg} / \mathrm{ml}$. After that, the sol was diluted 
with chloroform in a volume ratio of 1:4. We spread different amount of particles on water surface, and - after evaporation of the spreading liquid - compressed the monolayer film with a movable barrier, with a speed of $33.5 \mathrm{~cm}^{2} / \mathrm{min}$. Initial area of the water surface was $225 \mathrm{~cm}^{2}$, and the final area was $40 \mathrm{~cm}^{2}$. Wetting properties of fluorescent nanoparticles were evaluated according to the surface pressure-area isotherms; the characteristic parameters (collapse pressure, $\Pi_{c}$; collapse area, $A_{c}$; and contact cross-sectional area, $A_{k}$; see Fig. 1) were determined at room temperature. The water contact angles were calculated based on a model described previously [16, 17]. Reproducibility of making Langmuir films was also investigated; we spread a selected amount of particles on water surface three times, and compared characteristic parameters of the parallel isotherms.

\subsubsection{Preparation and characterization of monolayer}

\section{Langmuir-Blodgett (LB) films}

We deposited fluorescent nanoparticles on a glass substrate. The film transfer was carried out in four steps. First, the substrate was immersed into distilled water, after that, the silica particles were spread on water surface, the silica monolayer was compressed subsequently with a speed of $18.8 \mathrm{~cm}^{2} / \mathrm{min}$, and in the final step - reaching $50 \%$ of the collapse pressure of the sample - the substrate was slowly educed from the water. During the deposition, the surface pressure was kept constant. After finishing the procedure, we dried the Langmuir-Blodgett films at room temperature.

To demonstrate the presence of the deposited layer on the glass substrate, we made UV-Visible spectroscopy measurements before and after the film transfer. Transmittance spectra were determined at a wavelength range of 190-1100 nm.

We also measured the fluorescence of the film. Emission spectrum was determined at an excitation wavelength of $400 \mathrm{~nm}$, with an emission slit of $10 \mathrm{~nm}$. The higher value of the emission slit was set for increasing the signal/noise ratio. For the measurement, we used a $515 \mathrm{~nm}$ emission filter in order to eliminate noise that occurs due to reflection effects of the glass. Structure characterization was carried out with a Zeiss Evo 40 XVP scanning electron microscope (SEM). Prior to SEM measurements, the LB film was coated with a thin gold layer. Micrographs were made with 20000 times magnification. Energy of the beam was set to $20.00 \mathrm{kV}$.

\subsubsection{Stability investigation}

The stability of silica alco- and hydrosols of the fluorescent particles (in a $3 \mathrm{ml}$ volume,) was investigated by sedimentation studies. The sedimentation was studied during 7 days. We have chosen characteristic properties, such as height of the sedimentation front, which we defined as the distance between the solid phase border-line and zero height (see Fig. 2), normalized to the mean particle size $(\mathrm{mm})$, and the rate of sedimentation, which we defined as the quotient of the height of the front and the elapsed time (mm/day). Each day we measured the height of the sedimentation front, and compared the rate of sedimentation in hydro- and alcosols.

\section{Results}

\subsection{Accumulation of Thioflavin $\mathrm{T}$ molecules in silica nanoparticles}

Fig. 3 shows absorbance spectra of the dye solution, and the supernatant after separating it from the sediment.

According to calculations, the volume filled by silica particles was $6.0 \%$ of the total mixture volume. After 1 and 2 days, no accumulation was detectable. According to spectroscopy measurements, approximately $7.8 \%$ of the added dye was taken by the particles after one week. This quantity corresponds to a dye concentration of $1.03 * 10^{-8} \mathrm{~mol} / \mathrm{ml}$. This implies a slow accumulation process of the dye molecules into the native silica.

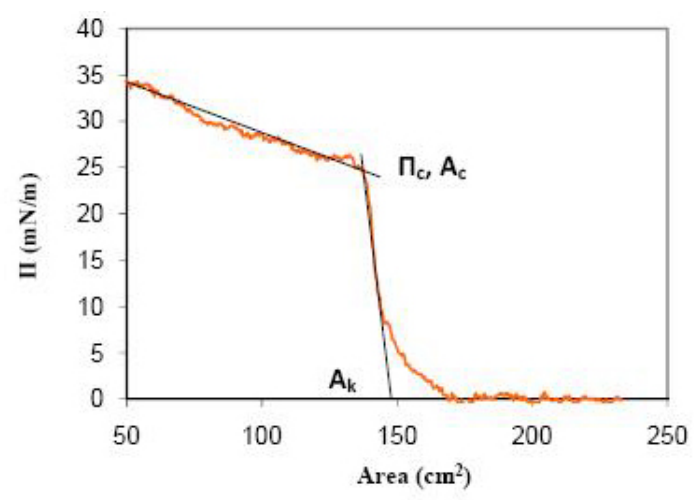

Fig. 1. Surface pressure-area isotherm of a Stöber silica nanoparticulate Langmuir film to determine characteristic parameters: collapse pressure ( $c)$, collapse area $(A c)$ and contact cross-sectional area $(A k)$.

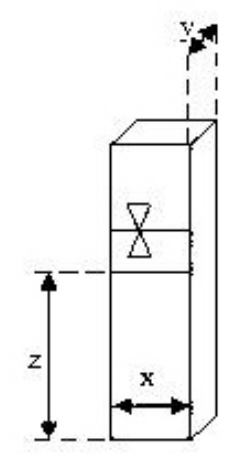

Fig. 2. Sol sedimentation in a cuvette. $X$ and $y$ represent width and depth of the cuvette, $z$ represents the height of the sedimentation front, and contrary triangles signify the liquid meniscus.

It is well known, that Stöber silica particles contain micropores [18], which could alter accumulation mechanisms. Based on a simplified monolayer adsorption model, estimating $94.24 \AA^{2}$ area for a single molecule2 (adsorbed parallel to the surface), and approximating the whole silica surface area on the basis of the mean diameter of silica spheres, we calculated the maximum amount of Thioflavin $\mathrm{T}$ on the geometric surface of particles. The resultant value $\left(2.57 \cdot 10^{-7} \mathrm{~mol} / \mathrm{ml}\right)$ was ca. 20 times higher than the experimentally determined one 


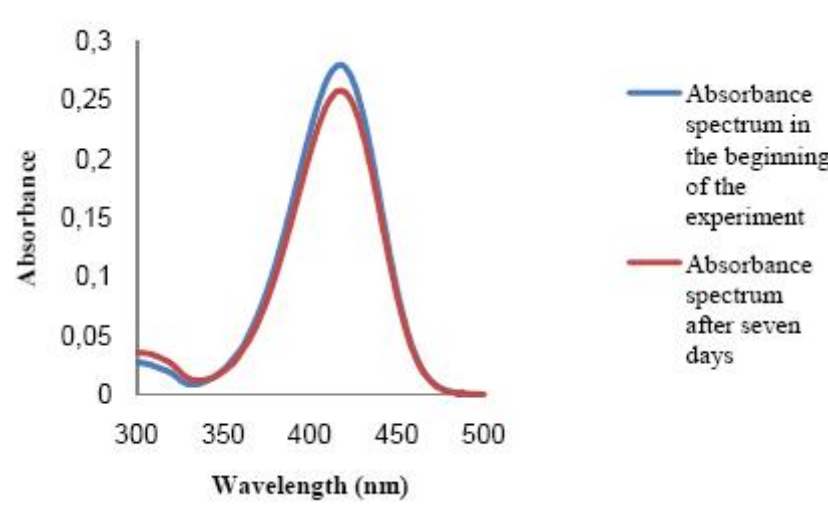

Fig. 3. Absorbance spectra of the starting dye solution, and the supernatant after separating it from the sediment one week later.

$\left(1.03 \cdot 10^{-8} \mathrm{~mol} / \mathrm{ml}\right)$. It means significant amount of dye cannot diffuse into the interior part of the particles after the investigated time interval if we suppose significant adsorption of the cationic dye at the surface of the particles.

\subsection{Release of Thioflavin T molecules from silica particles}

In purified alco- and hydrosols, presence of Thioflavin $\mathrm{T}$ in the supernatant was not detectable via UV-Vis spectrometry in any investigated case after two weeks. Therefore we assume that the prepared Stöber silica nanoparticles only release negligible quantity of the dye, which does not exceed the limit of detection, or the speed of release is quite slow.

\subsection{Particle size distribution}

The mean size of the particles and the standard deviation values are reported in Table 2, Fig. 4 shows TEM image of sample FB300E.

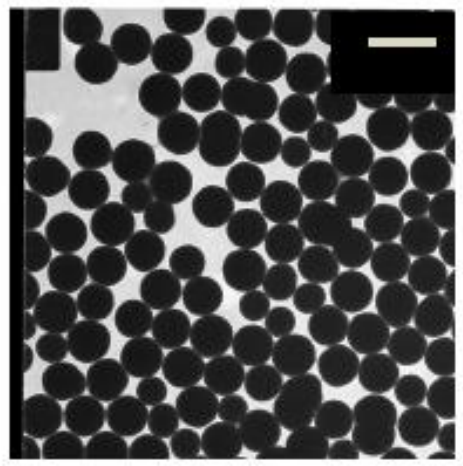

Fig. 4. TEM image of fluorescent nanoparticles FB300E. Scale bar represents $500 \mathrm{~nm}$

\subsection{Stability investigation}

We observed that the particles settled rather slowly with a diffuse sedimentation zone, both in ethanol and water demonstrating aggregative stability of samples. The resultant sedimentation volumes (shown in Table 3), which were reached after one week, do not show significant differences in different media.

Average sedimentation rate of the sols was approximately 20 $\mathrm{mm} /$ day, which means the prepared sols are stable, considering the particle sizes.

Thus we can say that both alco- and hydrosols of the prepared fluorescent silica particles are stable. Sols did not aggregate after heat shock, visually there was no change in stability.

\subsection{Fluorimetry measurement}

Intensity of emitted fluorescence was measurable in purified silica alcosols, thus we have evidenced, that the fluorescent dye was successfully integrated into the particles. Maxima of the sols' emission spectra (ca. 610units) were more than ten times higher than the highest emission intensity in ethanol solutions of Thioflavin $\mathrm{T}$ (approximately 22units) (see Figure 5a and 5b). Excitation spectrum of sol FB300B is showed in Figure 5c.

The excitation spectrum showed that highest emission intensity can be achieved with an excitation wavelength of $440 \mathrm{~nm}$. Sols FB300E and FB300F showed measurable fluorescence at solid content values of 2.4 and $1.2 \mathrm{mg} / \mathrm{ml}$. We suppose that fluorescence intensity was amplified due to an increase of the quantum yield, which was a consequence of the intercalation mechanisms of Thioflavin $\mathrm{T}$ molecules in silica nanoparticles. This intercalation mechanism with beta sheet proteins was reported previously [2]. According to fluorescence emission spectra, the method we used to synthesize fluorescent sols is reproducible.

Emission maxima of the sols reached a limit: above an amount of added dye, fluorescence intensity did not increase.

Tab. 3. Resultant sedimentation volumes of fluorescent silica alcosols after one week (in $3 \mathrm{ml}$ sol volume)

\begin{tabular}{ll}
\hline Sol & Sedimentation volume $\left(\mathrm{cm}^{3}\right)$ \\
\hline FB300B & 0.4 \\
FB300C & 0.3 \\
FB300D & 0.4 \\
FB300E & 0.4 \\
\hline
\end{tabular}

\subsection{Characterization of Langmuir films}

Fig. 6a shows surface pressure - area isotherms of different amounts of silica particles spread. Since contact angle must be independent from the amount of spread particles, and the pressure gradient between the moving barrier and the force meter has to be eliminated, extrapolation is needed. After extrapolation, contact angle can be determined [19]. Data of contact angles vs. the amount of particles spread and the trend line of extrapolation are shown in Fig. 6 p.

Contact angle of fluorescent silica nanoparticles with a mean diameter of $284 \mathrm{~nm}$ was found to be $18^{\circ}$, which meets our expectations that come from studies made previously, investigating silica particles with a diameter range of $45-100 \mathrm{~nm}$, using techniques like film balance and scanning angle reflectometry (these contact angles had been found to be in a range of $28^{\circ}$ $19^{\circ}$ ) [7|. Therefore, the surface of the fluorescent nanoparticles is very similar to that of as-prepared silica samples. This finding does not reveal significant adsorption of dye molecules at the 

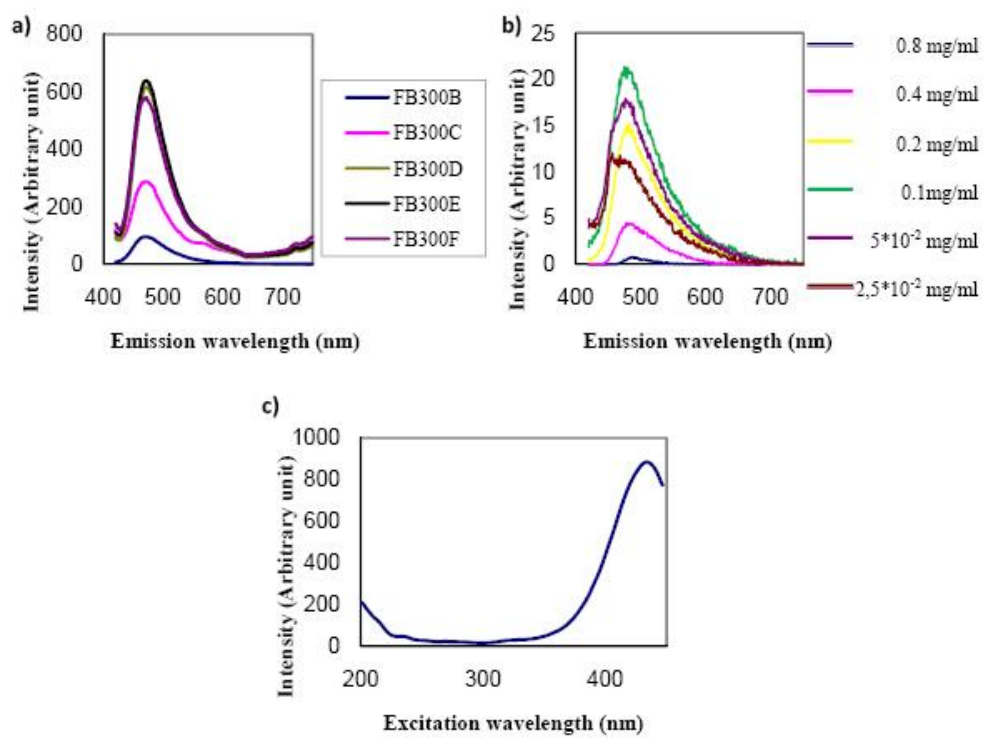

Fig. 5. Fluorescent spectra of silica sols: Emission spectra of Thioflavin $\mathrm{T}$ doped silica sols with solid content set to $12.0 \mathrm{mg} / \mathrm{ml}$, excited at $400 \mathrm{~nm}$ (a); Emission spectra of alcohol solutions of Thioflavin $\mathrm{T}$ with different dye concen-

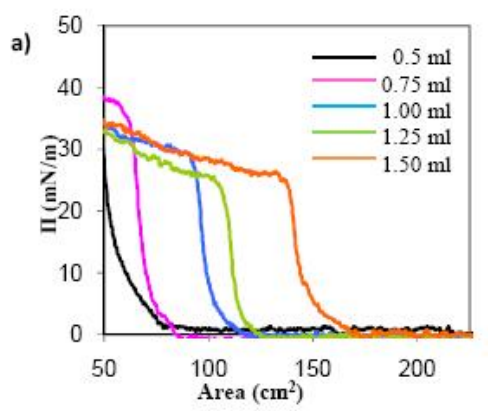

Fig. 6. Characterization of Langmuir films: Surface pressure - area isotherms of different particle amounts (a); Contact angle values for different surface of the particles and confirms our establishment that dye molecules accumulated in the inner part of particles.

Fig.7 7 shows the results of the reproducibility test. Preparation of Langmuir films showed to be reproducible, as the standard deviation of the parallel parameters' values was below $5 \%$.

\section{Characterization of monolayer LB films}

As shown in Figure 8, transmittance spectrum of the substrate after coating the particles differs from the transmittance spectrum measured before the deposition of the LB film. Thus, the presence of the Langmuir-Blodgett layer of silica particles was unambiguously revealed by the spectrum.

Fluorimetry measurements showed, that monolayer of $284 \mathrm{~nm}$ particles does not emit measurable fluorescent light, when excited at $400 \mathrm{~nm}$.

Fig. 9 shows scanning electron micrograph taken of the LB film of the sol FB300E.

According to the scanning electron micrograph, the particles formed a contiguous, well-packed monolayer on the support. tration, excited at $400 \mathrm{~nm}$ (b); Excitation spectrum of FB300B silica sol, with $480 \mathrm{~nm}$ emission wavelength (c).

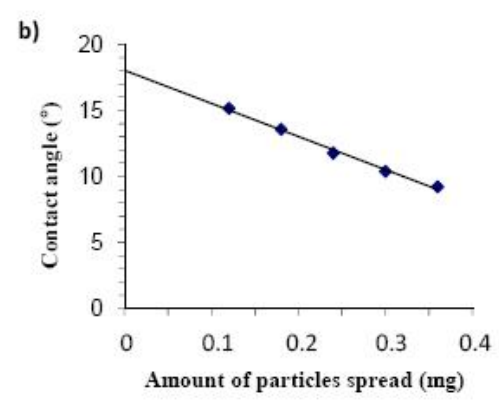

amount of particles spread, and the trend line of extrapolation (b).

\section{Summary}

The objective of this study was to synthesize and characterize Thioflavin T doped Stöber silica nanoparticles to establish novel signaling probes for medical applications, since the advantages of these two substances have not been combined before.

We investigated size distribution, morphology, fluorescent and wetting properties of the prepared particles, and also focused attention on the stability of their sols in water and ethanol phases. Furthermore, Langmuir and Langmuir-Blodgett films of the fluorescent silica nanospheres were prepared.

With fluorimetry investigations we evidenced that Thioflavin $\mathrm{T}$ molecules were integrated into the silica spheres, since the sols' fluorescence emission was measurable after the purification steps. In case of the silica sols, fluorescence intensity was more than ten times higher than in case of ethanol phase solutions of Thioflavin $\mathrm{T}$ conforming the localization of dye molecules in the silica matrix in a special conformation. According to TEM measurements, the particles $(284 \mathrm{~nm}$ average diameter) showed to be nearly monodisperse and spherical, no aggregation processes were observed.

We assessed the wettability of fluorescent particles from the non-dissipative part of surface pressure vs. surface area 
isotherms of Langmuir films. The resultant water contact angles $\left(18^{\circ}\right)$ showed that there is not significant amount of dye molecules at the surface of particles hence the fluorescent properties can be attributed to the intercalated dye molecules.

Adsorption investigations did not result in taking up Thioflavin $\mathrm{T}$ molecules into the interior part of native silica particles (prepared without fluorescent dye) during a week. The fluorescent particles, however, did not release fluorescent dye during the investigated time period which is required for the marking application.

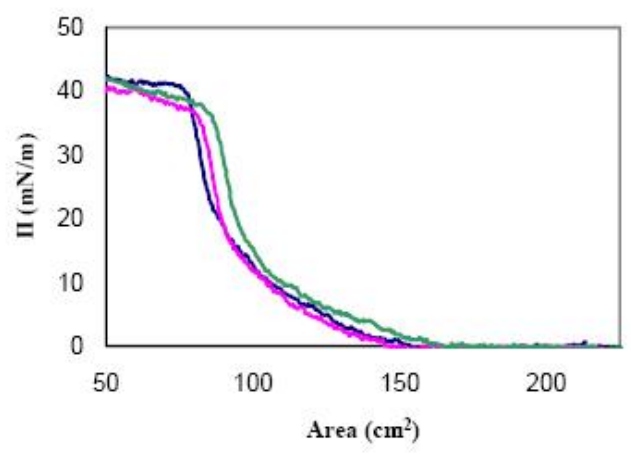

Fig. 7. Surface pressure-area isotherms of parallel measurements for the reproducibility test of establishing Langmuir films

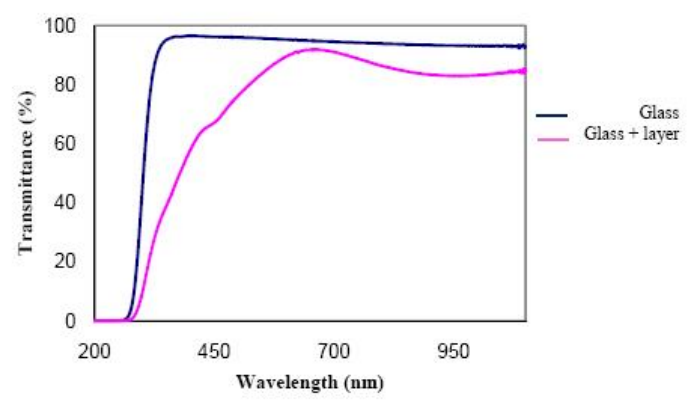

Fig. 8. Transmittance spectra of the glass substrate and the LB film coated glass substrate

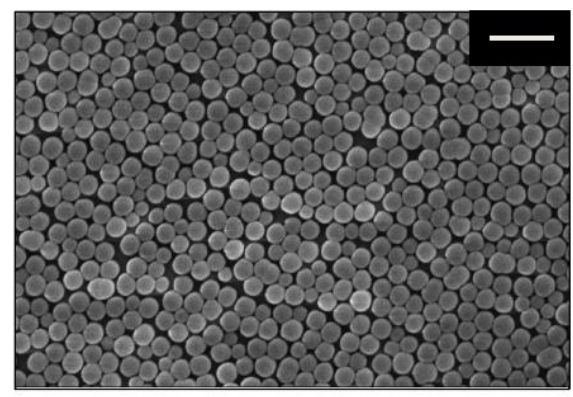

Fig. 9. Scanning electron micrograph taken of the Langmuir-Blodgett film of the sol FB300E. Scale bar represents $1 \mu \mathrm{m}$

\section{References}

1 Voropai E S, Samtsov M P, Kaplevskii K N, Maskevich A A, Stepuro V I, Kuznetsova I M, Turoverov K K, Fink A L, Uverskii V N, Spectral Properties of Thioflavin $T$ and Its Complexes with Amyloid Fibrils, Journal of Applied Spectroscopy 70 (2003), 868-874, DOI 10.1023/B:JAPS.0000016303.37573.7e.

2 Krebs M R H, Bromley E H C, Donald A M, The binding of thioflavin-T to amyloid fibrils: localisation and implications, Journal of Structural Biology 149 (2005), 30-37, DOI 10.1016/j.jsb.2004.08.002.
3 Pardridge W M, Transport of nutrients and hormones through the bloodbrain barrier, Diabetologia 20 (1981), 246-254, DOI 10.1007/BF00254490.

4 Mohanraj V J, Chen Y, Nanoparticles - A review, Trop. J. Pharm .Res. 5 (2006), 561-573.

5 Pathak P, Katiyar V K, Cancer Research - Nanoparticles, Nanobiosensors and Their Use in Cancer Research, Journal of Nanotechnology Online 3 (2007), DOI 10.2240/azojono0116.

6 van Vlerken L E, Amiji M M, Multi-functional polymeric nanoparticles for tumour-targeted drug delivery, Expert Opin. Drug Deliv. 3 (2006), 205-216, DOI 10.1517/17425247.3.2.205.

7 Siegemund T, Paulke B-R, Schmiedel H, Bordag N, Hoffmann A, Harkany T, Tanila $\mathbf{H}$, Kacza J, Härtig W, Thioflavins released from nanoparticles target fibrillar amyloid $\beta$ in the hippocampus of APP/PS1 transgenic mice, Int. J. Devl Neuroscience 24 (2006), 195-201, DOI 10.1016/j.ijdevneu.2005.11.012.

8 Härtig W, Paulke B R, Varga Cs, Seeger J, Harkany T, Kacza J, Electron microscopic analysis of nanoparticles delivering thioflavin-T after intrahippocampal injection in mouse: implications for targeting $\beta$-amyloid in Alzheimer's disease, Neuroscience Letters 338 (2003), 174-176, DOI 10.1016/S0304-3940(02)01399-X.

9 Stöber W, Fink A, Bohn E, Controlled growth of monodisperse silica spheres in the micron size range, J. Colloid Interface Sci. 26 (1968), 62-69, DOI 10.1016/0021-9797(68)90272-5.

10 Nakamura M, Shono M, Ishimura K, Synthesis, Characterization, and Biological Applications of Multifluorescent Silica Nanoparticles, Analytical Chemistry 79 (2007), 6507-6514, DOI 10.1021/ac070394d.

11 van Blaaderen A, Vrij A, Synthesis and characterization of colloidal dispersions of fluorescent, monodisperse silica spheres, Langmuir 8 (1992), 2921-2931, DOI 10.1021/la00048a013.

12 Verhaegh N A M, van Blaaderen A, Dispersions of RhodamineLabeled Silica Spheres: Synthesis, Characterization, and Fluorescence Confocal Scanning Laser Microscopy, Langmuir 10 (1994), 1427-1438, DOI 10.1021/la00017a019.

13 Deák A, Székely I, Kálmán E, Keresztes Zs, Kovács A L, Hórvölgyi Z, Nanostructured silica Langmuir-Blodgett films with antireflective properties prepared on glass substrates, Thin Solid Films 484 (2005), 310-317, DOI 10.1016/j.tsf.2005.01.096.

14 Deák A, Bancsi B, Tóth A L, Kovács A L, Hórvölgyi Z, Complex Langmuir-Blodgett films from silica nanoparticles: An optical spectroscopy study, Colloids and Surfaces A: Physicochem. Eng. Aspects 278 (2006), 1016, DOI 10.1016/j.colsurfa.2005.11.070.

15 Santra S, Liesenfeld B, Bertolino C, Dutta D, Cao Z, Tan W, Moudgil B M, Mericle R A, Fluorescence lifetime measurements to determine the core-shell nanostructure of FITC-doped silica nanoparticles: An optical approach to evaluate nanoparticle photostability, Journal of Luminescence 117 (2006), 75-82, DOI 10.1016/j.jlumin.2005.04.008.

16 Deák A, Hild E, Kovács A L, Hórvölgyi Z, Contact angle determination of nanoparticles: film balance and scanning angle reflectometry studies, Physical Chemistry Chemical Physics 9 (2007), 6359-6370, DOI 10.1039/b702937n.

17 Bordács S, Agod A, Hórvölgyi Z, Composed of Fine Particles: Collapse Mechanism and Wettability, Langmuir 22 (2006), 6944-6950, DOI 10.1021/la060696v.

18 Lecloux A J, Bronckart J, Noville F, Dodet C, Marchot P, Pirard J P, Study of the texture of monodisperse silica sphere samples in the nanometer size range, Colloids and Surfaces 19 (1986), 359-374, DOI 10.1016/01666622(86)80289-X.

19 Máté M, Fendler J H, Ramsden J R, Szalma J, Hórvölgyi Z, Eliminating Surface Pressure Gradient Effects in Contact Angle Determination of Nanoand Microparticles Using a Film Balance, Langmuir 14 (1998), 6501-6504, DOI 10.1021/la971344e. 